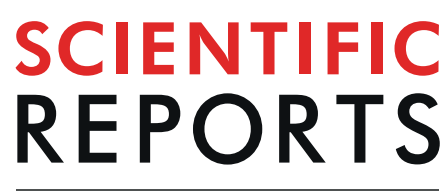

natureresearch

Received: 14 May 2019

Accepted: 20 September 2019

Published online: 07 October 2019

\section{Corticostriatal stimulation compensates for medial frontal inactivation during interval timing}

\author{
Eric B. Emmons $\mathbb{D}^{1}$, Morgan Kennedy ${ }^{2}$, Youngcho $\mathrm{Kim}^{3}$ \& Nandakumar S. Narayanan ${ }^{3}$
}

Prefrontal dysfunction is a common feature of brain diseases such as schizophrenia and contributes to deficits in executive functions, including working memory, attention, flexibility, inhibitory control, and timing of behaviors. Currently, few interventions improve prefrontal function. Here, we tested whether stimulating the axons of prefrontal neurons in the striatum could compensate for deficits in temporal processing related to prefrontal dysfunction. We used an interval-timing task that requires working memory for temporal rules and attention to the passage of time. Our previous work showed that inactivation of the medial frontal cortex (MFC) impairs interval timing and attenuates ramping activity, a key form of temporal processing in the dorsomedial striatum (DMS). We found that 20-Hz optogenetic stimulation of MFC axon terminals increased curvature of time-response histograms and improved interval-timing behavior. Furthermore, optogenetic stimulation of terminals modulated time-related ramping of medium spiny neurons in the striatum. These data suggest that corticostriatal stimulation can compensate for deficits caused by MFC inactivation and they imply that frontostriatal projections are sufficient for controlling responses in time.

The prefrontal cortex is dysfunctional in psychiatric disorders such as schizophrenia ${ }^{1,2}$ and neurodegenerative disorders such as Huntington's disease and Parkinson's disease ${ }^{3,4}$. Prefrontal impairments are associated with executive dysfunction, including disruption of working memory, attention, flexibility, reasoning, and timing of behavioral responses ${ }^{5}$. Currently, few interventions can mitigate or compensate for prefrontal dysfunction in human disease.

Prefrontal neurons send axons to the basal ganglia ${ }^{6-9}$, and prefrontal impairments can disrupt the function of neurons in subcortical areas ${ }^{10}$. In the present study, we investigated whether manipulating the striatum could compensate for deficits in prefrontal function. To answer this question, we used interval timing, which assesses the ability of subjects to estimate an interval of several seconds based on a motor response. Interval timing is highly translational because it involves both prefrontal and striatal regions in rodents as well as in humans and other primates ${ }^{11-14}$ and the neuronal correlates in frontostriatal circuits are similar across species ${ }^{15-17}$. In addition, interval timing is relevant to our research question because it is reliably disrupted in human diseases that impair prefrontal function ${ }^{16-20}$.

A recent study from our group demonstrated that pharmacological inactivation of the rodent medial frontal cortex (MFC; dorsal prelimbic + anterior cingulate) ${ }^{21,22}$ impaired interval timing and attenuated a key neuronal correlate of temporal processing in the dorsomedial striatum (DMS): time-related ramping activity ${ }^{13,23}$. These data indicate that temporal processing by DMS neurons requires input via MFC axons, and predict that stimulating $\mathrm{MFC} \rightarrow$ DMS axonal projections can compensate for deficits in temporal control of action as well as striatal temporal processing in animals with the MFC inactivated.

We tested this idea by inactivating the MFC and optogenetically stimulating the terminals of MFC axons in the DMS while rodents performed a 12-second (s) fixed-interval timing task. In animals with intact MFC function, optogenetic stimulation of MFC axon terminals in the DMS had few consistent effects. By contrast, in MFC-inactivated animals, optogenetic stimulation of MFC $\rightarrow$ DMS terminals improved response times, curvature of time-response histograms, and time-dependent ramping by DMS neurons. We interpret our results in the context of top-down frontal control of striatal activity, which could be relevant for efforts to mitigate prefrontal dysfunction. 
A

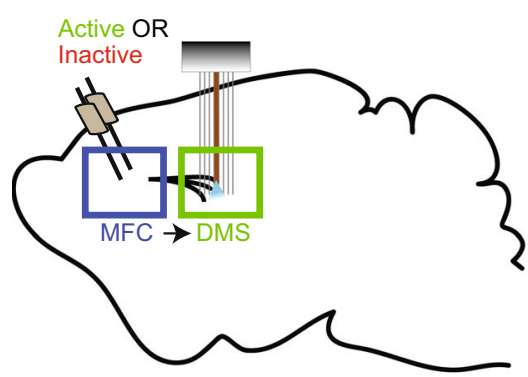

C

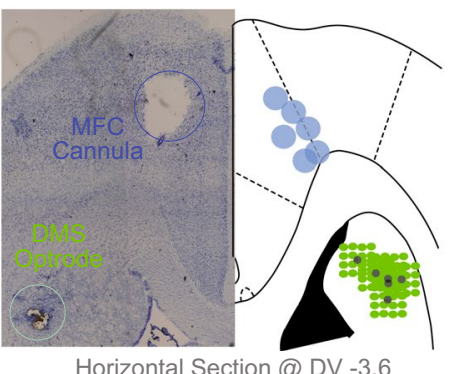

B

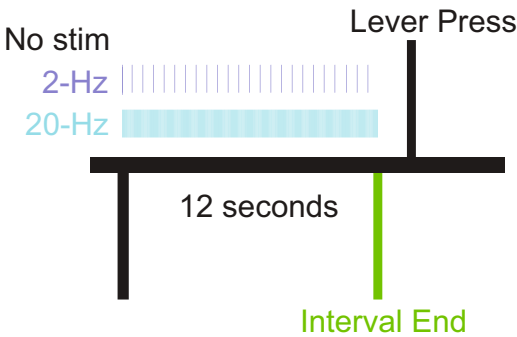

$\mathbf{D}$

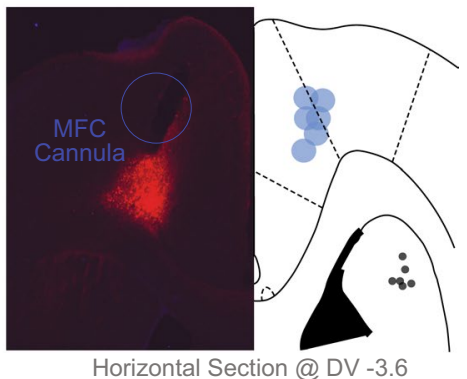

Figure 1. Interval-timing task paradigm and histology. (A) Depiction of the surgical approach for the combined optogenetic stimulation and electrophysiological recording experiments. Both MFCs were injected with AAV-ChR2 (or control AAV-mCherry without opsins), after which they were implanted with cannulae along with DMS optrodes (multielectrode recording arrays surrounding a fiber optic cannula). (B) Schematic of interval-timing task. A houselight cue signaled the onset of a trial. $12 \mathrm{~s} \mathrm{later,} \mathrm{a} \mathrm{reward} \mathrm{was} \mathrm{available} \mathrm{in} \mathrm{response}$ to a lever press. MFC (DMS axons were optogenetically stimulated at $2 \mathrm{~Hz}$ or $20 \mathrm{~Hz}$ during the interval. (C) Left: Representative image of histology in the left hemisphere showing MFC cannula and DMS optrode tracts. Right: Histological reconstruction of placement of cannulae and optrode in 6 AAV-ChR2-injected animals. (D) Left: Histology of the left hemisphere of a control animal showing MFC cannula and expression of viral mCherry in the MFC and DMS fiber optics. Right: Histological reconstruction of placement of cannulae and fiber optics in 6 control AAV-mCherry-injected animals.

\begin{tabular}{|l|l|l|}
\hline $\begin{array}{l}\text { Observations: } \mathbf{8 9 2 5} \\
\mathbf{R}^{\mathbf{2}} \mathbf{0 . 0 1 3}\end{array}$ & FStat & p-value \\
\hline Stim & 0.21 & 0.8 \\
\hline Drug & 2.9 & 0.09 \\
\hline Stim:Drug & 5.2 & 0.006 \\
\hline vs. Null model & LR: 19.4 & 0.002 \\
\hline
\end{tabular}

Table 1. Trial-by-Trial GLMM of response times.

\section{Results}

Optogenetic stimulation of MFC axons in the DMS normalizes interval-timing behavior. We investigated whether corticostriatal stimulation could compensate for MFC inactivation in 12 rats performing a 12-s interval-timing task. In these rats, both sides of the MFC had been injected with AAV-CamKIIa-ChR2 (MFC-ChR2; Fig. 1) or a control virus, AAV-mCherry. Animals were also implanted with bilateral MFC infusion cannulae. After the rats were acclimatized to infusion and recording procedures, both hemispheres of the MFC were bilaterally infused with either saline or the $\mathrm{GABA}_{\mathrm{A}}$ agonist muscimol, which reversibly and completely inactivates the $\mathrm{MFC}^{13,24,25}$. Following this infusion, all animals performed interval timing under three different laser stimulation parameters: no stimulation (No Stim), 2- Hz stimulation (2-Hz), or 20-Hz stimulation $(20-\mathrm{Hz})$. Critically, stimulation was unilateral whereas inactivation was bilateral. We used a generalized linear mixed-effects model (GLMM) to capture effects of optogenetic stimulation on response times in sessions during which the MFC was inactive. In AAV-CamKIIa-ChR2 animals, this analysis revealed a significant interaction between MFC inactivation and optogenetic stimulation on response time $(F=5.2, p=0.006$; see Table 1 for stats; Fig. 2A,B). This interaction was not observed in control animals with AAV-mCherry (interaction: $F=0.9, p=0.41$ ).

We also quantified interval-timing performance based on the 'curvature' of time-response histograms (Fig. 2C-E) ${ }^{26,27}$. In line with our past work, MFC inactivation with muscimol decreased the curvature of time-response histograms relative to saline sessions $\left(0.26 \pm 0.02\right.$ vs. $0.12 \pm 0.02$; paired $t_{(11)}=3.5, p=0.01$; Fig. 2D). In support of our hypothesis, we found that $20-\mathrm{Hz}$ optogenetic stimulation of the terminals of MFC axons within the DMS increased the curvature of time-response histograms when the MFC was inactivated in 

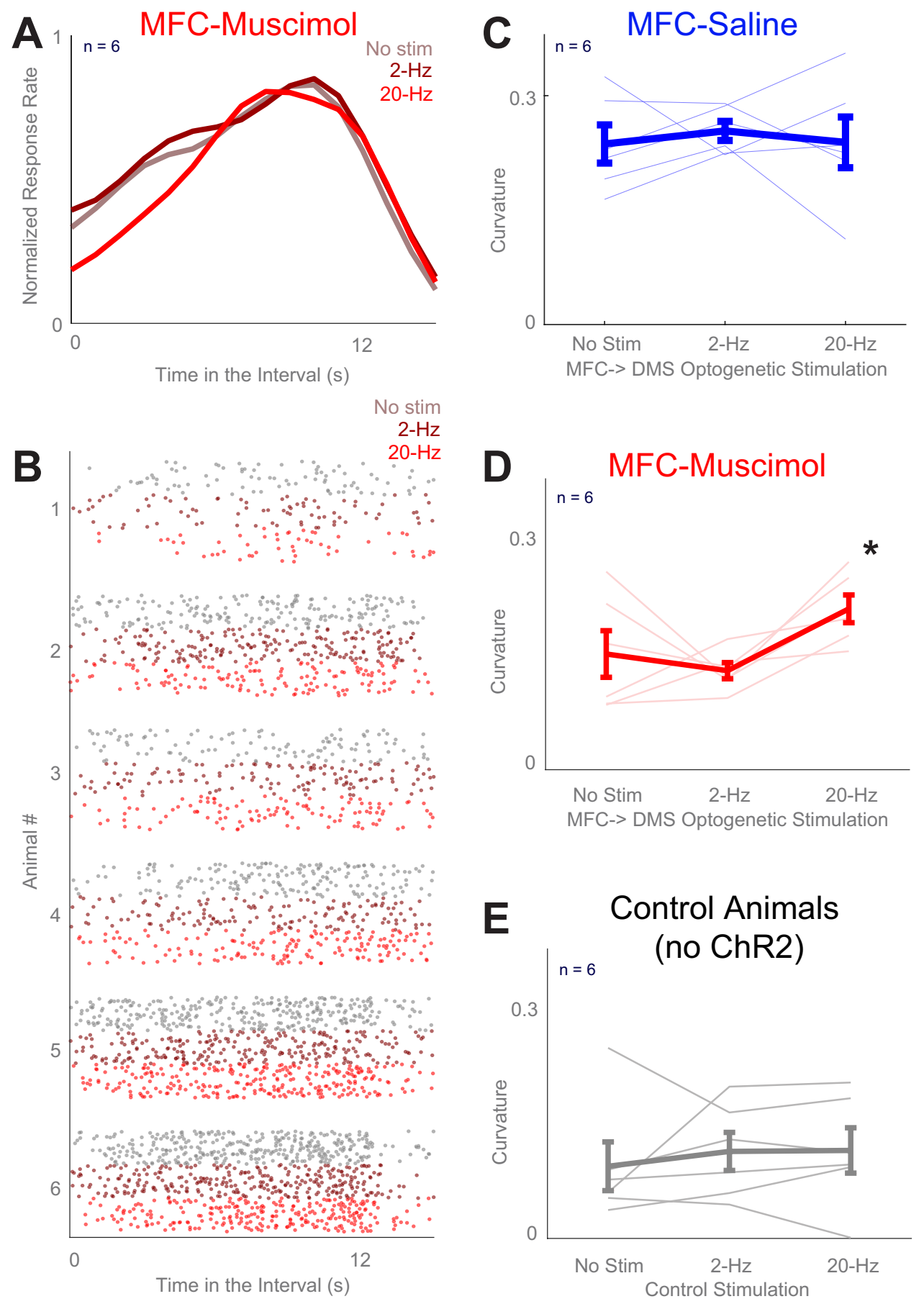

Figure 2. Stimulation of terminals of MFC $\rightarrow$ DMS axons influences the timing of responses during interval timing. (A) Average time-response histograms of behavior across laser stimulation conditions. We tested three laser stimulation conditions: No Stim (grey), 2-Hz (dark red), or 20-Hz (bright red). (B) Raster plots showing all responses of each animal in the study during interval-timing behavior following infusion of the MFC with muscimol (same colors as in A). (C) Average curvature values for animals across stimulation condition for MFC-Saline and (D) for MFC-Muscimol sessions across stimulation conditions; C and D are from the same 6 animals expressing AAV-CamIIK-ChR2 on separate infusion days. (E) Average curvature values for control animals across stimulation conditions in the MFC-Muscimol session; data from 6 separate animals expressing AAV-CamKII-mCherry. All data represent mean \pm SEM from sessions in which MFC was inactivated using muscimol; $* p<0.05$.

6 animals with AAV- CamKIIa -ChR2 (Fig. 2 D; $0.15 \pm 0.03$ vs. $0.21 \pm 0.02$; paired $t_{(5)}=2.6, p=0.05$; Cohen's $d=0.95$ ). No significant effects were noted for $2-\mathrm{Hz}$ stimulation, in animals with the MFC active, or in control animals with AAV-mCherry (Fig. 2D-E). A GLMM modeling effects of optogenetic stimulation and MFC 
A

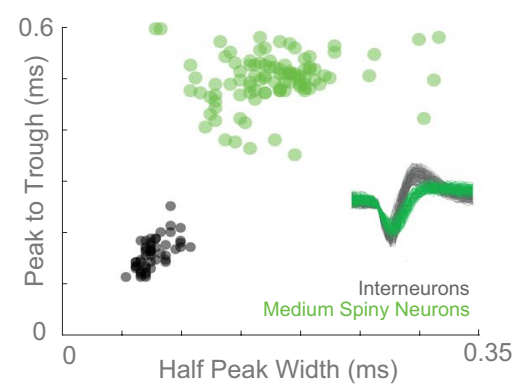

B

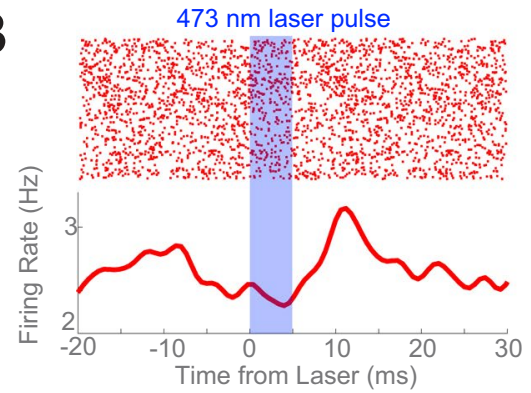

C

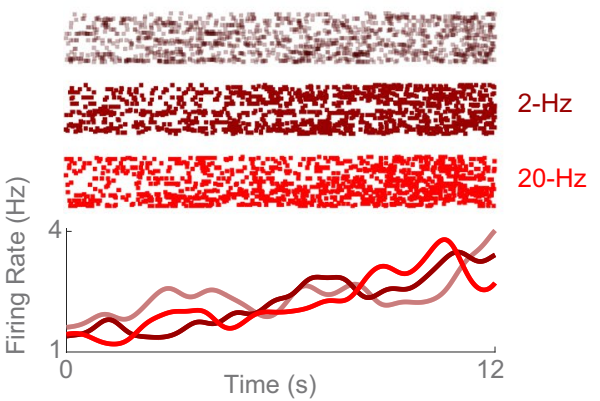

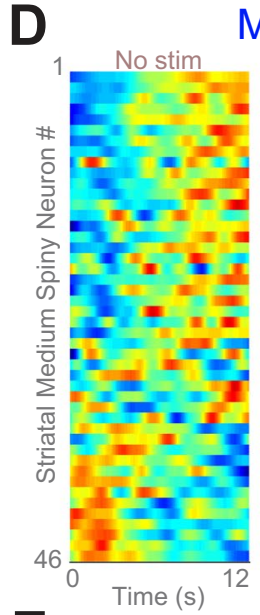

MFC-Saline
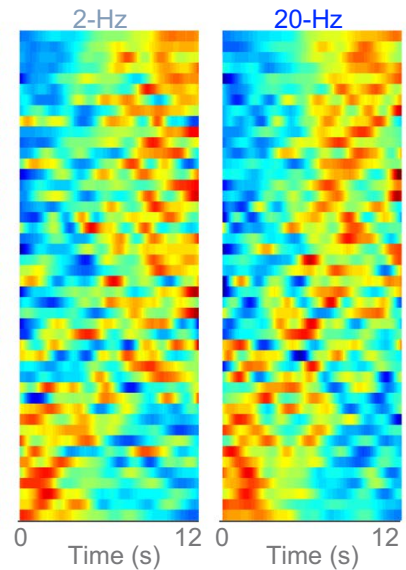

$\mathbf{E}$

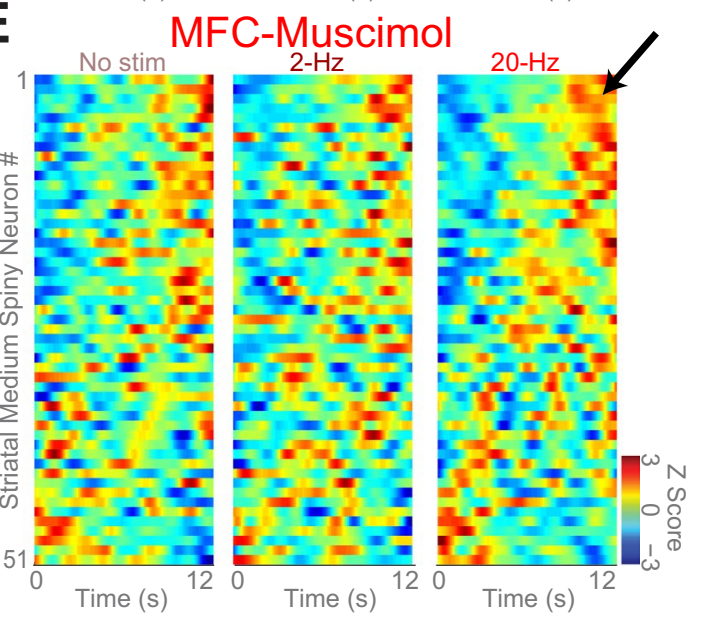

Figure 3. Analysis of MSNs in DMS of animals performing the interval-timing task. (A) Separation of medium spiny neurons (MSNs; green) from interneurons (black) in the dorsomedial striatum (DMS) based on peak-totrough duration and half-peak-width; $\mathrm{ms}=$ millisecond. (B) An MSN with increased firing after MFC-DMS axonal stimulation; note that this recording occurred with muscimol infused into MFC. (C) An exemplar DMS MSN exhibiting increased time-related ramping with $20-\mathrm{Hz}$ stimulation of $\mathrm{MFC} \rightarrow$ DMS axons; this neuron was recorded during MFC-Muscimol infusion. (D) Peri-event time histograms showing normalized firing rate of all MSNs within the DMS in MFC-Saline sessions and in (E) MFC-Muscimol sessions under each laser stimulation condition. Neurons are sorted based on the first principal component, which, as in our past work, is a ramping pattern of activity. Red indicates higher firing rates whereas blue indicates lower firing rate. Robust ramping was observed in MFC-Saline sessions; black arrow highlights increased number of positively-ramping neurons with $20-\mathrm{Hz}$ stimulation.

inactivation on curvature revealed a main effect of MFC inactivation on curvature $(F=8.9, p=0.006)$ and a trend towards an interaction between MFC inactivation and optogenetic stimulation $(F=2.6, p=0.09)$. These data trend towards a dose-dependent response of MFC $\rightarrow$ DMS axonal stimulation in MFC-inactivation sessions.

To further analyze interval-timing behavior, we turned to single-trial analysis ${ }^{28}$. This analysis was developed for peak-interval-timing behavior to measure when rats start and stop responding; however, because we did a fixed-interval task we only analyzed the start times from our data. We found that MFC inactivation shifted the start times earlier in the interval $\left(7.8 \pm 0.3 \mathrm{~s}\right.$ with muscimol vs $8.4 \pm 0.2 \mathrm{~s}$ with saline; paired $\left.t_{(5)}=2.6, p=0.05\right)$. By contrast, $20-\mathrm{Hz}$ MFC $\rightarrow$ DMS axonal stimulation shifted start times later in the interval ( $8.8 \pm 0.4 \mathrm{~s}$; paired $\left.t_{(5)}=2.8, p=0.04\right)$. Together, these data provide evidence that optogenetic stimulation of corticostriatal axons at $20 \mathrm{~Hz}$ influenced the temporal control of responses primarily when the MFC was inactive.

Optogenetic stimulation of MFC axons in DMS alters time-dependent ramping in DMS MSNs. Our recent work demonstrated that inactivating the MFC impaired interval timing and attenuated ramping activity in the DMS. Here, we tested the hypothesis that stimulation of MFC axons in the DMS increased time-dependent ramping of DMS neurons. We investigated this question by recording from DMS medium spiny neurons (MSNs; Fig. 3A) while inactivating the MFC and stimulating MFC $\rightarrow$ DMS axons in the 6 animals with AAV-CamKIIa-ChR2. Because the number of fast-spiking interneurons identified by our recording approach was only half that of MSNs, we focused our analyses on MSNs. 


\begin{tabular}{|l|l|l|}
\hline $\begin{array}{l}\text { Observations: } \mathbf{1 5 5 6 4 0 0 0} \\
\mathbf{R}^{2} \mathbf{0 . 0 1 1}\end{array}$ & FStat & p-value \\
\hline Times & 333.7 & $1.5 e^{-74}$ \\
\hline Stim & 8.3 & 0.0003 \\
\hline Drug & 0.001 & 0.97 \\
\hline Times:Stim & 1.1 & 0.34 \\
\hline Times:Drug & 35.6 & $2.5 e^{-9}$ \\
\hline Stim:Drug & 5.5 & 0.004 \\
\hline Times:Stim:Drug & 11.4 & 0.00001 \\
\hline vs. Null model & LR: 30526 & $\sim 0$ \\
\hline
\end{tabular}

Table 2. Trial-by-Trial GLMM of MSN firing rate.

We used a GLMM to analyze the effects of MFC inactivation and optogenetic stimulation on DMS firing (See Table 2). As in our past work, we found that DMS firing rates were affected by time in the interval (i.e., time-related ramping; $\left.F=333.7, p=1.5 \mathrm{e}^{-74}\right)$ and an interaction between MFC inactivation and firing rates over time $\left(F=35.6, p=2.5 \mathrm{e}^{-9}\right)^{13}$. MFC axons comprise $\sim 10 \%$ of the input to striatal MSNs; accordingly, we found no main effect of MFC inactivation on DMS firing. MFC axons release glutamate, and we found a main effect of MFC $\rightarrow$ DMS axonal stimulation on DMS firing $(F=8.3, p=0.0003)$ that interacted with MFC inactivation $(F=5.5$, $p=0.004)$. Furthermore, we found that DMS neurons could be modulated by MFC $\rightarrow$ DMS axonal stimulation (Fig. 3B).

Crucially, the GLMM indicated that there was an interaction between time, optogenetic stimulation, and MFC inactivation $(F=11.4, p=0.00001$; Table 2$)$. These data indicate that optogenetic stimulation changes time-dependent ramping activity as a function of MFC inactivation. This insight was supported by peri-event time histograms which revealed increased time-dependent ramping on trials with $20-\mathrm{Hz}$ optogenetic stimulation of MFC $\rightarrow$ DMS axons relative to $2-\mathrm{Hz}$ or No-Stim trials in MFC-inactivation sessions (Fig. 3C,E). Such changes were not observed in MFC-Saline sessions (Fig. 3D). We also examined the slope of MSN ramping neurons in MFC-inactivation sessions. The slope was highest on trials with $20-\mathrm{Hz} \mathrm{MFC} \rightarrow \mathrm{DMS}$ axonal stimulation $\left(0.05 \pm 0.01\right.$ on $20-\mathrm{Hz}$ trials vs. $0.03 \pm 0.01$ on No-Stim trials, paired $t_{(50)}=2.5, p=0.01$, Cohen's $d=0.26$; when restricted to neurons that exhibited ramping: $0.06 \pm 0.01$ on $20-\mathrm{Hz}$ trials vs. $0.04 \pm 0.01$ on No-Stim trials; paired $\left.t_{(38)}=2.1, p=0.05\right)$. These analyses provide evidence that the interaction between time-dependent ramping, optogenetic stimulation, and MFC inactivation observed in the GLMM analysis was due primarily to increased time-dependent ramping on trials with $20-\mathrm{Hz}$ optogenetic stimulation. Taken together, our results suggest that optogenetic stimulation of MFC axons in the DMS changed both response times and time-dependent ramping activity of DMS neurons as a function of MFC inactivation.

\section{Discussion}

We tested the hypothesis that stimulation of corticostriatal projections could compensate for temporal-processing deficits caused by prefrontal inactivation. We found that in animals in which the MFC was inactivated, stimulation of $\mathrm{MFC} \rightarrow$ DMS axons at $20 \mathrm{~Hz}$ - but not $2 \mathrm{~Hz}$-could normalize the curvature of time-response histograms and shift response times during interval timing. Furthermore, we found that optogenetic stimulation of MFC $\rightarrow$ DMS axons interacted with DMS time-dependent ramping activity as a function of MFC inactivation, tending to increase the slope of DMS ramping activity with $20-\mathrm{Hz}$ stimulation in MFC inactivation sessions. Our results provide novel evidence that monosynaptic projections from the MFC to the DMS are sufficient to compensate for behavioral deficits in interval timing caused by MFC inactivation, and also suggest that these projections modulate temporal processing in the DMS.

MFC neurons are involved in top-down control of goal-directed behavior ${ }^{29-31}$. Consistent with our prior work, we found that MFC inactivation decreased the number of MSNs within the DMS that exhibited time-dependent ramping activity, implying that without input from the MFC, a key correlate of temporal processing in the DMS was attenuated. We found that optogenetic stimulation of MFC $\rightarrow$ DMS axons could interact with both time-dependent ramping activity and MFC inactivation, providing evidence that stimulation of these axons was sufficient to modulate time-related ramping in MSNs. However, we did not find that optogenetic stimulation increased the number of neurons in the DMS that exhibited time-dependent ramping. Our effect sizes, particularly in GLMMs, were small. However, our intervention was relatively restricted in that stimulation was unilateral but pharmacological inactivation was bilateral ${ }^{32,33}$. Whereas MFC inactivation reversibly and completely silences MFC networks $s^{25,34,35}$, our optogenetic approach likely stimulated only a small fraction of MFC axons due to the unilateral manipulation and the limitations of viral expression. Furthermore, corticostriatal projections to the striatum are highly organized and specific. Our optogenetic approach nonspecifically stimulated MFC axons. Finally, the activity of MFC neurons during interval timing is highly dynamic ${ }^{13}$, while our stimulation was constant across the interval. In light of these caveats, it is remarkable that the stimulation of corticostriatal terminals was sufficient to influence interval-timing behavior by shifting response times and increasing the curvature of time-response histograms. These data suggest that control of the MFC over DMS ensembles occurs via direct, monosynaptic projections rather than being an indirect effect of MFC projections to another area that modulate the $\mathrm{DMS}^{8,9}$.

Here we found stimulation effects at $20 \mathrm{~Hz}$, whereas our past work stimulating MFC networks or MFC afferents revealed effects primarily in the delta/theta range $(\sim 4 \mathrm{~Hz})^{16,17,36,37}$. Cortical stimulation can be resonant with 
$\sim 4-\mathrm{Hz}$ activity among prefrontal networks involved in cognitive control ${ }^{37}$. The striatum may have distinct network properties from cortex. Corticostriatal projections release glutamate; we found main effects of MFC axonal optogenetic stimulation on DMS firing that interacted with MFC inactivation. Our data are consistent with a role for these glutamatergic projections modulating time-related ramping as $20-\mathrm{Hz}$ stimulation likely would release more glutamate at corticostriatal synapses than $2-\mathrm{Hz}$ stimulation ${ }^{38}$. Interneurons can also be modulated by corticostriatal projections, although we recorded fewer such cells, and we did not find consistent evidence for inhibition of MSN activity by MFC $\rightarrow$ DMS stimulation ${ }^{39}$. Consequently, we focused our analyses on MSNs.

Our study has several additional limitations. Firstly, our approach was limited by the sampling of only a small number of DMS MSNs, and by the nonspecific, virally-mediated expression of opsin in MFC axons that project to the striatum. DMS ramping may depend on specific projections and/or patterns of activity. Additionally, our recording approach did not allow us to differentiate between D1- and D2-dopamine receptor-expressing MSNs or various subtypes of interneurons, and such distinctions might be key to temporal processes ${ }^{40-42}$. Indeed, our recent pharmacological work has suggested that these neuronal populations may perform in distinct ways during interval-timing tasks ${ }^{43}$ and MFC stimulation may preferentially affect one class of neurons over others. Our experiments were designed based on fixed-interval timing; however, future experiments using a peak-interval task may enable us to capture additional temporal computations. Furthermore, we did find acute effects of optogenetic stimulation on MSN firing, but our experiments were not designed to definitively map corticostriatal circuits. Alternative designs with moveable arrays in the striatum may achieve this aim. Finally, the work presented here was restricted to interval timing, and likely needs to be expanded, both to other stimulation parameters as well as to other tasks involving sensorimotor and cognitive processing.

In summary, our experiments provide novel evidence that corticostriatal circuits play a critical role in accurate information processing. Combined with our prior work demonstrating that MFC activity is necessary for temporal processing in the DMS, this work suggests that MFC $\rightarrow$ DMS projections may be sufficient for some level of rescue of deficits in interval-timing behavior caused by prefrontal dysfunction. We anticipate that these data will inform clinical therapies that target corticostriatal glutamate ${ }^{44,45}$ or deep-brain stimulation of the striatum for disorders that affect the prefrontal cortex ${ }^{46}$.

\section{Methods}

Rodents. All procedures were approved by the University of Iowa IACUC and all methods were performed in accordance with the relevant guidelines and regulations (protocol \#7072039). Twelve male Long-Evans rats were trained on an interval-timing task as described in detail previously ${ }^{13,25,36,47}$. Briefly, animals were initially autoshaped to press a lever for water reward using a fixed-ratio task. Then, animals were trained on a 12-s fixed-interval task (FI12). Trials began with the presentation of a houselight at trial onset (time 0), and the first response made after $12 \mathrm{~s}$ had passed resulted in reward delivery, a concurrent click sound, and termination of the houselight (Fig. 1B). Responses made before $12 \mathrm{~s}$ elapsed had no programmed consequence. Trials were separated by a randomly chosen intertrial interval of $6,8,10$ or $12 \mathrm{~s}$. Sessions lasted 60 minutes $(\mathrm{m})$. The timing of each response was used to compute average response rate as a function of time within a trial.

Surgical and histological procedures. The MFCs of twelve rats were bilaterally infused with AAVs and then implanted with bilateral infusion cannulae. Six of the animals were injected with AAVs that express channelrhodopsin (ChR2); the other six animals were injected with AAVs expressing mCherry and no active ChR2. The left dorsomedial striatum (DMS) of 6 ChR2 animals was implanted with fiber optic recording electrodes (referred to here as "optrodes", which were implanted unilaterally). A surgical level of anesthesia was maintained and, under aseptic surgical conditions, craniotomies were drilled above the left and right MFCs as well as the left DMS. Rat MFCs were first injected with AAV-CamKII-mCherry-ChR2 (AAV-ChR2) or AAV-CamKII-mCherry (AAV-mCherry) virus (1.0 uL virus per side; UNC Viral Vector Core, Chapel Hill, NC). Each rat was later implanted with bilateral infusion cannulae. The left DMS was implanted with a 16-wire optrode (microelectrode array combined with fiber optic cannula). In addition, we used four skull screws. The ground wire from the optrode was connected to the screws and passed into the brain. Optrode arrays consisted of $1650-\mu \mathrm{m}$ stainless steel wires arranged in two concentric circles of eight wires surrounding the fiber optic cannula $(250 \mu \mathrm{m}$ between wires and rows; impedance measured in vitro at $\sim 400 \mathrm{k} \Omega$; Microprobes for Life Science; Gaithersburg, MD). Infusion cannulae targeted both MFCs (coordinates from bregma: AP + 3.2, ML $\pm 1.2, \mathrm{DV}-3.6 @ 12^{\circ}$ in the anterior plane; these coordinates target the dorsal prelimbic cortex), whereas the optrode recording array targeted only the left DMS (coordinates from bregma: AP + 0.0, ML $\pm 4.2, \mathrm{DV}-3.6 @ 12^{\circ}$ in the lateral plane). Optrode arrays were inserted while recording neuronal activity in order to verify that the implant correctly targeted the DMS. The craniotomy was sealed with cyanoacrylate ('SloZap', Pacer Technologies, Rancho Cucamonga, CA), whose polymerization was accelerated by 'ZipKicker' (Pacer Technologies); and with methyl methacrylate (i.e., dental cement; AM Systems, Port Angeles, WA). Following implantation, the animals were given one week to recover before being reacclimatized to behavioral and recording procedures.

Following completion of the behavioral experiments, the rats were anesthetized and sacrificed by injection of $100 \mathrm{mg} / \mathrm{kg}$ sodium pentobarbital and transcardially perfused with $4 \%$ formalin. Brains were post-fixed in a solution of $4 \%$ formalin and $20 \%$ sucrose before being sectioned on a freezing microtome. Brain slices were mounted on gelatin-coated slides and cell bodies were identified by staining with either DAPI or Cresyl violet. For each animal, histological reconstruction was completed based on postmortem analysis of electrode placements by confocal microscopy or stereology microscopy. These data were used to determine the locations of the electrodes and cannulae within the MFC, and that of the electrode in the DMS (Fig. 1C). Immunohistochemistry was used to visualize the expression of AAV-CamKII-mCherry-ChR2 and AAV-CamKII-mCherry. 
Protocol for rodent behavioral experiments. Rats were first trained in the fixed interval-timing task (FI12) and then underwent stereotactic surgery, as described above. Animals were given one week to recover before being acclimatized to recording and/or stimulation procedures. To ensure that viral expression was maximal, experiments began 3-4 weeks after surgery. Electrophysiological recordings and/or optogenetic stimulation were performed on subsequent days. Infusions of saline and muscimol were performed on separate days. On the first day of the infusion protocol, animals received bilateral saline infusions through both cannulae in the MFC. On the second day, animals were infused with the $\mathrm{GABA}_{\mathrm{A}}$ receptor agonist muscimol $(0.1 \mathrm{mg} / \mathrm{mL}, 0.5 \mu \mathrm{L})$, an approach we have used previously to reversibly and completely inhibit cortical neuronal activity ${ }^{13,25}$. In all recording experiments and/or drug infusions, each session was treated as statistically independent ${ }^{13,24,25,47-49}$. Following the infusions, animal behavior on the FI12 interval-timing task was compared between various conditions of optogenetic stimulation with a $473-\mathrm{nm}$ laser (5-ms pulse width, $10 \mathrm{~mW}$ power; Opto Engine, Midvale, UT). Three different stimulation conditions were used in each animal: no stimulation (No Stim), 2- $\mathrm{Hz}$ stimulation (2-Hz), and $20-\mathrm{Hz}$ stimulation $(20-\mathrm{Hz}$; Fig. 1A). Conditions were pseudo-randomly interleaved such that the number of trials of each condition $(+/-1$ trial) was equal. Behavioral sessions lasted for $60 \mathrm{~m}$.

Protocol for neurophysiological recordings and analyses of neurons. Neuronal ensemble recordings were made using a multi-electrode recording system (Plexon, Dallas, TX). Putative single neuronal units were identified on-line using an oscilloscope and an audio monitor (Plexon, Dallas, TX). Plexon Offline Sorter was used to analyze the signals after the experiments were completed, and to remove artifacts. Spike activity was analyzed for all cells that fired at rates above $0.1 \mathrm{~Hz}$. Principal component analysis (PCA) and waveform shape were used to sort spikes. Analysis of neuronal activity and quantitative analysis of basic firing properties were carried out using the NeuroExplorer software (Nex Technologies, Littleton, MA) and custom routines available in the MATLAB suite. In each animal, one electrode with minimal neuronal activity was reserved for local referencing, so that 15 electrodes per animal were available for spiking activity. Putative neurons were classified as either medium spiny neurons (MSNs) or fast-spiking interneurons based on waveform peak-to-trough ratio and half-peak widths ${ }^{50}$. MSNs were identified from these parameters by Gaussian mixture clustering in MATLAB (fitgmdist.m). Because significantly fewer fast-spiking interneurons were identified, we restricted our analyses to MSNs.

Statistics. In accordance with our prior work, we quantified temporal control of action by calculating the curvature of time-response histograms. Curvature values range between -1 and 1 and are calculated from the measured cumulative response record by its deviation from a straight line, where 0 would indicate a constant response rate throughout the interval. Curvature indices are resistant to differences in response rate, smoothing, or binning ${ }^{26,27}$.

To quantify the effects of optogenetic stimulation on behavior on a trial-by-trial basis, we used generalized linear mixed-effects models (GLMM; fitglme in MATLAB). To quantify the effects of optogenetic stimulation and MFC inactivation on behavior, we used the following GLMM to quantify response time:

$$
\mathrm{RT} \sim \operatorname{Stim} * \text { Drug }+(1 \mid \text { Animal })
$$

where the dependent variable was response time $(R T)$, independent variables were optogenetic stimulation (Stim: No Stim, 2-Hz Stim, or 20-Hz Stim) and MFC inactivation (Drug), and animals were included as a random variable (1|Animal). We ran separate models for AAV-ChR2 animals and for AAV-mCherry control animals. All GLMMs were compared with the null model via the function compare. $m$. For plotting only, kernel density estimates of time-response histograms were calculated. $k$ sdensity. $m$ in MATLAB was used with a bandwidth of 1 , normalized to the maximum response rate in each animal, and then averaged.

To quantify the effects of optogenetic stimulation, MFC inactivation, and the time of the interval on neuronal activity, we used the following GLMM to quantify neuronal firing rate:

$$
\mathrm{FR} \sim \text { Time } * \text { Stim } * \text { Drug }+(1 \mid \text { Neuron })
$$

where the dependent variable was firing rate ( $F R$; binned at $10 \mathrm{~ms}$ over the $12 \mathrm{~s}$ interval), and independent variables were time (Time over the $12 \mathrm{~s}$ interval in $10 \mathrm{~ms}$ bins), optogenetic stimulation (Stim: No Stim, 2-Hz Stim, or $20-\mathrm{Hz}$ Stim) and MFC inactivation (Drug); neurons were included as a random variable (1|Neuron).

For the analysis of individual neurons, we ran the GLMMs for each neuron of firing rate as a function of time in the interval or of stimulation. This analysis enabled us to identify effects and calculate regression slopes at the single-neuron level. In line with past work, we defined neurons for which the main effect of time had a $p<0.05$ as exhibiting time-dependent ramping activity. All regression analyses were performed on unsmoothed, unnormalized firing rate. For plotting only, peri-event rasters were binned over $10 \mathrm{~ms}$ and smoothed over $1 \mathrm{~s}$ with a Gaussian kernel; peri-event time histograms were binned at $100 \mathrm{~ms}$ and smoothed using ksdensity.m in MATLAB with a bandwidth of 0.5 , normalized using $\mathrm{z}$-scores, and sorted by the first principal component.

\section{Data Availability}

All data is available at https://narayanan.lab.uiowa.edu/datasets, and a pre-print version of this manuscript is available on BioRxiv.

\section{References}

1. Andreasen, N. C. et al. Hypofrontality in neuroleptic-naive patients and in patients with chronic schizophrenia. Assessment with xenon 133 single-photon emission computed tomography and the Tower of London. Arch. Gen. Psychiatry 49, 943-958 (1992).

2. Andreasen, N. C. et al. Hypofrontality in schizophrenia: distributed dysfunctional circuits in neuroleptic-naïve patients. Lancet 349, 1730-1734 (1997). 
3. Deutch, A. Y. Prefrontal cortical dopamine systems and the elaboration of functional corticostriatal circuits: implications for schizophrenia and Parkinson’s disease. J. Neural Transm. Gen. Sect. 91, 197-221 (1993).

4. Nopoulos, P. et al. Morphology of the cerebral cortex in preclinical Huntington's disease. Am. J. Psychiatry 164, 1428-1434 (2007).

5. Fuster, J. The Prefrontal Cortex, Fourth Edition. (Academic Press, 2008).

6. Alexander, G. E. \& Crutcher, M. D. Functional architecture of basal ganglia circuits: neural substrates of parallel processing. Trends Neurosci. 13, 266-271 (1990).

7. Ferry, A. T., Ongür, D., An, X. \& Price, J. L. Prefrontal cortical projections to the striatum in macaque monkeys: Evidence for an organization related to prefrontal networks. J. Comp. Neurol. 425, 447-470 (2000).

8. Gabbott, P. L. A., Warner, T. A., Jays, P. R. L., Salway, P. \& Busby, S. J. Prefrontal cortex in the rat: projections to subcortical autonomic, motor, and limbic centers. J. Comp. Neurol. 492, 145-177 (2005).

9. Han, S.-W., Kim, Y.-C. \& Narayanan, N. S. Projection targets of medial frontal D1DR-expressing neurons. Neurosci. Lett. 655, 166-171 (2017)

10. Meyer-Lindenberg, A. et al. Reduced prefrontal activity predicts exaggerated striatal dopaminergic function in schizophrenia. Nat. Neurosci. 5, 267-271 (2002).

11. Matell, M. S. \& Meck, W. H. Cortico-striatal circuits and interval timing: coincidence detection of oscillatory processes. Brain Res. Cogn. Brain Res. 21, 139-170 (2004)

12. Coull, J. T., Cheng, R.-K. \& Meck, W. H. Neuroanatomical and neurochemical substrates of timing. Neuropsychopharmacol. Off. Publ. Am. Coll. Neuropsychopharmacol. 36, 3-25 (2011).

13. Emmons, E. B. et al. Rodent Medial Frontal Control of Temporal Processing in the Dorsomedial Striatum. J. Neurosci. 37, 8718-8733 (2017).

14. Wang, J., Narain, D., Hosseini, E. A. \& Jazayeri, M. Flexible timing by temporal scaling of cortical responses. Nat. Neurosci. 21, 102 (2018).

15. Parker, K. L., Chen, K.-H., Kingyon, J. R., Cavanagh, J. F. \& Naryanan, N. S. Medial frontal 4 Hz activity in humans and rodents is attenuated in PD patients and in rodents with cortical dopamine depletion. J. Neurophysiology 114, 1310-1320 (2015).

16. Parker, K. L. et al. Delta-frequency stimulation of cerebellar projections can compensate for schizophrenia-related medial frontal dysfunction. Mol. Psychiatry 22, 647-655 (2017).

17. Kim, Y.-C. et al. Optogenetic Stimulation of Frontal D1 Neurons Compensates for Impaired Temporal Control of Action in Dopamine-Depleted Mice. Curr. Biol. CB 27, 39-47 (2017).

18. Buhusi, C. V. \& Meck, W. H. What makes us tick? Functional and neural mechanisms of interval timing. Nat. Rev. Neurosci. 6, 755-765 (2005).

19. Ward, R. D., Kellendonk, C., Kandel, E. R. \& Balsam, P. D. Timing as a window on cognition in schizophrenia. Neuropharmacology 123, 720-730 (2011).

20. Parker, K. L., Lamichhane, D., Caetano, M. S. \& Narayanan, N. S. Executive dysfunction in Parkinson's disease and timing deficits. Front. Integr. Neurosci. 7, 75 (2013).

21. Laubach, M. A comparative perspective on executive and motivational control by the medial prefrontal cortex. In Neural Basis of Motivational and Cognitive Control (MIT Press, 2011).

22. Laubach, M., Amarante, L. M., Swanson, K. \& White, S. R. What, If Anything, Is Rodent Prefrontal Cortex? eNeuro 5 (2018).

23. Simen, P., Balci, F., de Souza, L., Cohen, J. D. \& Holmes, P. A model of interval timing by neural integration. J. Neurosci. Off. J. Soc. Neurosci. 31, 9238-9253 (2011).

24. Narayanan, N. S., Horst, N. K. \& Laubach, M. Reversible inactivations of rat medial prefrontal cortex impair the ability to wait for a stimulus. Neuroscience 139, 865-876 (2006).

25. Parker, K. L., Chen, K.-H., Kingyon, J. R., Cavanagh, J. F. \& Narayanan, N. S. D1-Dependent 4 Hz Oscillations and Ramping Activity in Rodent Medial Frontal Cortex during Interval Timing. J. Neurosci. 34, 16774-16783 (2014).

26. Fry, W., Kelleher, R. T. \& Cook, L. A mathematical index of performance on fixed-interval schedules of reinforcement. J. Exp. Anal. Behav. 3, 193-199 (1960)

27. Narayanan, N. S., Land, B. B., Solder, J. E., Deisseroth, K. \& DiLeone, R. J. Prefrontal D1 dopamine signaling is required for temporal control. Proc. Natl. Acad. Sci. USA 109, 20726-20731 (2012).

28. Church, R. M., Meck, W. H. \& Gibbon, J. Application of scalar timing theory to individual trials. J. Exp. Psychol. Anim. Behav. Process. 20, 135-155 (1994).

29. Niki, H. \& Watanabe, M. Cingulate unit activity and delayed response. Brain Res. 110, 381-6 (1976).

30. Ostlund, S. B. \& Balleine, B. W. Lesions of medial prefrontal cortex disrupt the acquisition but not the expression of goal-directed learning. J. Neurosci. 25, 7763-70 (2005).

31. Ma, L., Hyman, J. M., Phillips, A. G. \& Seamans, J. K. Tracking progress toward a goal in corticostriatal ensembles. J. Neurosci. Off. J. Soc. Neurosci. 34, 2244-2253 (2014).

32. Xu, R. et al. Quantitative comparison of expression with adeno-associated virus (AAV-2) brain-specific gene cassettes. Gene Ther. 8 , 1323-1332 (2001).

33. Hommel, J. D., Sears, R. M., Georgescu, D., Simmons, D. L. \& DiLeone, R. J. Local gene knockdown in the brain using viralmediated RNA interference. Nat. Med. 9, 1539-1544 (2003).

34. Martin, J. H. \& Ghez, C. Pharmacological inactivation in the analysis of the central control of movement. J. Neurosci. Methods 86, 145-59 (1999).

35. Allen, T. A. et al. Imaging the spread of reversible brain inactivations using fluorescent muscimol. J. Neurosci. Methods 171, 30-38 (2008).

36. Emmons, E. B., Ruggiero, R. N., Kelley, R. M., Parker, K. L. \& Narayanan, N. S. Corticostriatal Field Potentials Are Modulated at Delta and Theta Frequencies during Interval-Timing Task in Rodents. Front. Psychol. 7 (2016).

37. Kim, Y.-C. \& Narayanan, N. S. Prefrontal D1 Dopamine-Receptor Neurons and Delta Resonance in Interval Timing. Cereb. Cortex N. Y. N 1991 29, 2051-2060 (2018).

38. Kreitzer, A. C. Physiology and Pharmacology of Striatal Neurons. Annu. Rev. Neurosci. 32, 127-147 (2009).

39. Burguiere, E., Monteiro, P., Feng, G. \& Graybiel, A. M. Optogenetic Stimulation of Lateral Orbitofronto-Striatal Pathway Suppresses Compulsive Behaviors. Science 340 (2013).

40. Wall, N. R., De La Parra, M., Callaway, E. M. \& Kreitzer, A. C. Differential Innervation of Direct- and Indirect-Pathway Striatal Projection Neurons. Neuron 79, 347-360 (2013).

41. Tecuapetla, F., Matias, S., Dugue, G. P., Mainen, Z. F. \& Costa, R. M. Balanced activity in basal ganglia projection pathways is critical for contraversive movements. Nat. Commun. 5 (2014).

42. Howard, C. D., Li, H., Geddes, C. E. \& Jin, X. Dynamic Nigrostriatal Dopamine Biases Action Selection. Neuron 93, 1436-1450.e8 (2017).

43. De Corte, B. J., Wagner, L. M., Matell, M. S. \& Narayanan, N. S. Striatal dopamine and the temporal control of behavior. Behav. Brain Res. 356, 375-379 (2019).

44. Emre, M. et al. Memantine for patients with Parkinson's disease dementia or dementia with Lewy bodies: a randomised, doubleblind, placebo-controlled trial. Lancet Neurol. 9, 969-977 (2010).

45. Garcia-Munoz, M., Lopez-Huerta, V. G., Carrillo-Reid, L. \& Arbuthnott, G. W. Extrasynaptic glutamate NMDA receptors: key players in striatal function. Neuropharmacology 89, 54-63 (2015). 
46. Dougherty, D. D. et al. A Randomized Sham-Controlled Trial of Deep Brain Stimulation of the Ventral Capsule/Ventral Striatum for Chronic Treatment-Resistant Depression. Biol. Psychiatry 78, 240-248 (2015).

47. Parker, K. L., Ruggiero, R. N. \& Narayanan, N. S. Infusion of D1 Dopamine Receptor Agonist into Medial Frontal Cortex Disrupts Neural Correlates of Interval Timing. Front. Behav. Neurosci. 9, 294 (2015).

48. Narayanan, N. S., Cavanagh, J. F., Frank, M. J. \& Laubach, M. Common medial frontal mechanisms of adaptive control in humans and rodents. Nat. Neurosci. 16, 1888-1897 (2013).

49. Narayanan, N. S. \& Laubach, M. Neuronal correlates of post-error slowing in the rat dorsomedial prefrontal cortex. J. Neurophysiol. 100, 520-525 (2008).

50. Berke, J. D., Okatan, M., Skurski, J. \& Eichenbaum, H. B. Oscillatory Entrainment of Striatal Neurons in Freely Moving Rats. Neuron 43, 883-896 (2004).

\section{Author Contributions}

E.B.E. and N.S.N. designed experiments, E.B.E. collected data, E.B.E., M.K., Y.K. and N.S.N. analyzed data, and E.B.E., Y.K. and N.S.N. wrote the paper.

\section{Additional Information}

Competing Interests: The authors declare no competing interests.

Publisher's note Springer Nature remains neutral with regard to jurisdictional claims in published maps and institutional affiliations.

Open Access This article is licensed under a Creative Commons Attribution 4.0 International License, which permits use, sharing, adaptation, distribution and reproduction in any medium or format, as long as you give appropriate credit to the original author(s) and the source, provide a link to the Creative Commons license, and indicate if changes were made. The images or other third party material in this article are included in the article's Creative Commons license, unless indicated otherwise in a credit line to the material. If material is not included in the article's Creative Commons license and your intended use is not permitted by statutory regulation or exceeds the permitted use, you will need to obtain permission directly from the copyright holder. To view a copy of this license, visit http://creativecommons.org/licenses/by/4.0/.

(C) The Author(s) 2019 